\title{
Overexpression of the Granulocyte Colony-Stimulating Factor Gene Impairs Bone Morphogenetic Protein Responsiveness in Mice
}

\author{
Hiroki Kuwabara, Takuro Wada, Takashi Oda, Hideki Yoshikawa, \\ Norimasa Sawada, Yasuo Kokai, and Seiichi Ishii \\ Departments of Orthopedic Surgery (HK, TW, TO, SI) and Pathology (HK, NS, YK), Sapporo Medical University, \\ Sapporo; and Department of Orthopedic Surgery (HY), Osaka University, Osaka, Japan
}

SUMMARY: Granulocyte colony-stimulating factor (G-CSF) is the major hematopoietic growth factor regulating the production and differentiation of neutrophils. We previously demonstrated that permanent overexpression of G-CSF in transgenic mice produces a dramatic enlargement of the bone cavity and reduction of bone mass. This phenotype was shown to be associated with an increase of osteoclast-mediated bone resorption. As a way of determining the role of G-CSF in bone formation in vivo, an ectopic bone was induced subcutaneously into G-CSF transgenic mice by bone morphogenetic protein (BMP)-2, a potent initiator of bone and cartilage from undifferentiated mesenchymal cells. A BMP-2/atelocollagen pellet containing recombinant human BMP-2 was implanted into a dorsal subfascial pocket. At one week after implantation, proliferation of mesenchymal cells around the implant was significantly decreased in transgenic mice compared with control mice. At three weeks, an ectopic bone containing bone marrow was formed both in transgenic and control mice. However, the ectopic bones of the transgenic mice were smaller and less consistent than those of control mice, and the calcium contents were reduced to $56.2 \%$ of those of controls. The ectopic bone in the G-CSF mice showed poor development of both lamellar and trabecular bone. Semiquantitative reverse transcription-polymerase chain reaction analysis of the ectopic bone at 3 weeks disclosed no significant differences in the mRNA levels of type I collagen, osteopontin, and osteocalcin between G-CSF mice and control mice. Immunohistochemical study in G-CSF mice showed reduced staining of osteocalcin in the bone matrix surrounding the reduced number of osteoblasts. The half-life of BMP in the implants was prolonged to 7 to 9 days in the G-CSF mice, whereas it was 5 days in the control mice. Collectively, the permanent expression of G-CSF may retard the differentiation process of osteoblasts by impairing the initial induction of mesenchymal cells, resulting in reduction of bone mass, suggesting that G-CSF regulates the bone metabolism by modulating both osteoclast and osteoblast function. Furthermore, it is suggested that G-CSF is a potent modulator of the BMP-2 signal pathway in vivo. (Lab Invest 2001, 81:1133-1141).

G ranulocyte colony-stimulating factor (G-CSF) is the major hematopoietic growth factor regulating the production of neutrophils, and its recombinant form has been used in various clinical settings (Glaspy and Golde, 1992; To et al, 1997; Wada et al, 1996). Although the activities of G-CSF were believed to be restricted to cells of granulocytic lineage, it has been found that this cytokine has a biological activity in bone metabolism.

Long-term exposure to G-CSF has been reported to stimulate osteoclast-mediated bone resorption in human patients with congenital neutropenia (Bishop et al, 1995; Yakisan et al, 1997) and in normal rodents (Lee et al, 1991; Soshi et al, 1996). We previously demonstrated that permanent G-CSF overproduction in transgenic mice produces a dra-

\section{Received April 16, 2001.}

This work was supported by grants-in-aid from the Ministry of Education, Science, and Culture, from the Ministry of Health and Welfare of Japan, and from Akiyama Memorial Research Foundation.

Address reprint requests to: Dr. Takuro Wada, Department of Orthopedic Surgery, Sapporo Medical University, South-1 West-16, Sapporo 0608543 Japan. E-mail: twada@sapmed.ac.jp matic enlargement of the bone cavity and reduction of bone mass. Histomorphometric analysis revealed a significantly increased osteoclast surface and osteoclast number in the vertebra of G-CSF mice (Takahashi et al, 1996). These observations consistently suggest that G-CSF stimulates osteoclastmediated bone resorption.

In contrast, the effect of G-CSF on bone formation is still controversial. In humans, the serum osteocalcin concentration is strongly reduced after 3 days of G-CSF administration, suggesting inhibition of bone formation (Takamatsu et al, 1998). Conversely, the serum concentration in G-CSF transgenic mice is significantly higher than that of their littermate controls (Takahashi et al, 1996). In rats treated with recombinant human granulocyte colony-stimulating factor (rhG-CSF), accelerated osteogenesis, resulting from intramembranous ossification and bone resorption, was observed in the metaphyseal spongiosa (Suzuki et al, 1999).

As a way of determining the role that G-CSF plays in bone formation, ectopic bone formation was induced subcutaneously in G-CSF mice by bone morphoge 
netic protein (BMP)-2. Here, we demonstrate that the ectopic bone formation was significantly impaired in mutant mice. These observations suggest that G-CSF is a potent modulator of bone metabolism, not only by stimulating osteoclast-mediated bone resorption, but also by inhibiting osteoblast-mediated bone formation.

\section{Results}

\section{Macroscopic Findings on the Ectopic Bone Induced by BMP-2}

The macroscopic characteristics of the ectopic bone formation 3 weeks after implantation are shown in Figure 1. The ectopic bones in control mice were reddish in color and very hard. The ectopic bones in the G-CSF mice were smaller in size, with a pale color and less consistency.

\section{Rentogenographic Findings of the Ectopic Bone}

The radiological appearance of the ectopic bone 3 weeks after implantation is shown in Figure 2. In the littermate control, there were calcified trabeculae surrounded by thick peripheral calcification. In the G-CSF mice, however, the calcified trabeculae were less evident, and the peripheral calcification was thinner than in control mice (Fig. 2).
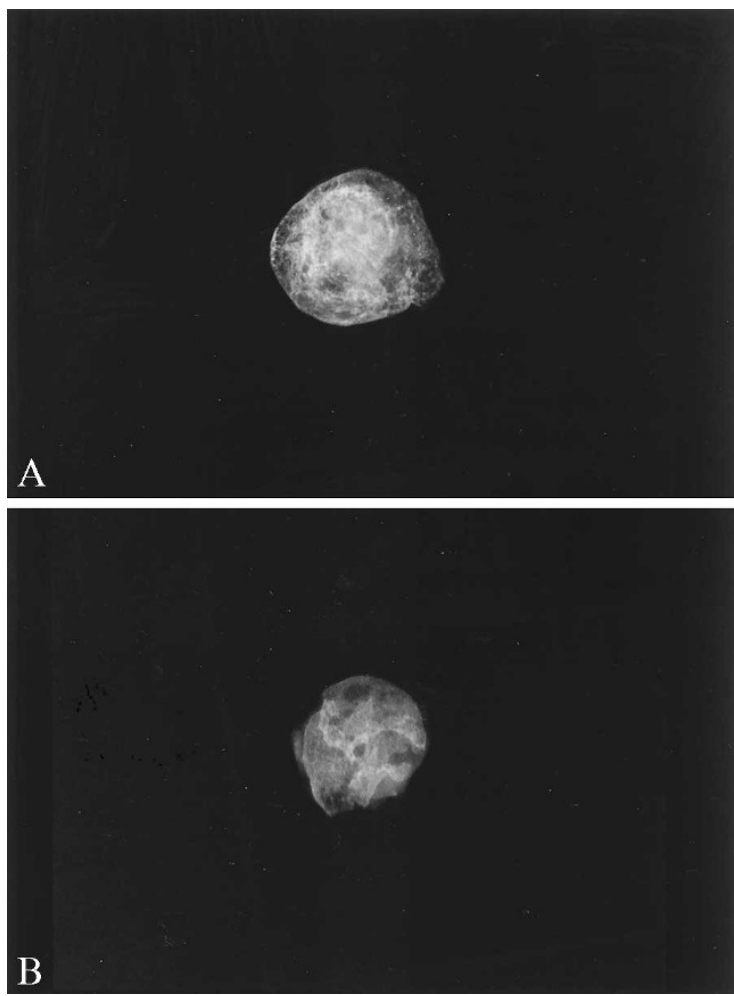

Figure 2.

Radiographs of the ectopic bone induced by BMP-2. Surface calcification and calcified trabeculae are clearly observed in the ectopic bone of a littermate control (A). Little calcification is seen in the ectopic bone of the mutant mice (B).

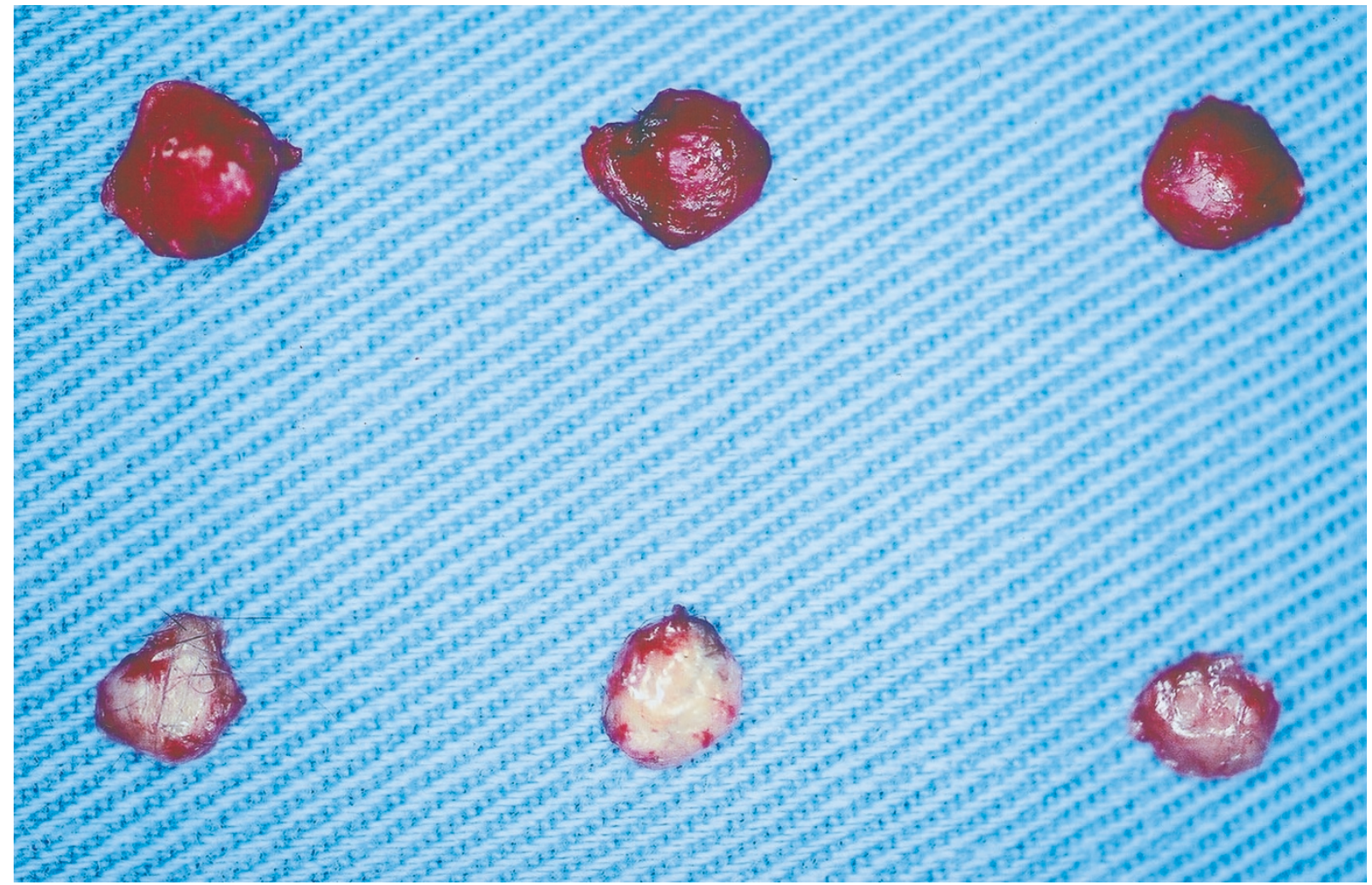

\section{Figure 1.}

Macroscopic findings on the ectopic bone induced by bone morphogenetic protein (BMP)-2. In the control mice (upper row), the color of the ectopic bones is reddish. The ectopic bones of the mutant mice (lower row) look white and are smaller than those of the littermate controls 


\section{Histological Examinations of the BMP-Pellets}

At 1 week, proliferation of mesenchymal cells surrounding the implants was observed in control mice (Fig. 3). There were islands of cartilage cells inside the implants. The size of the cartilage islands was smaller, and the number of the islands was fewer in mutant mice than in control mice. At 3 weeks, the maximum coronal section of the ectopic bone showed well-developed lamellar bone outside and clear trabeculae inside in control mice. The ectopic bone in the G-CSF mice showed poor development of both lamellar and trabecular bone. In addition, discontinuity of the lamellar structure was found in
A
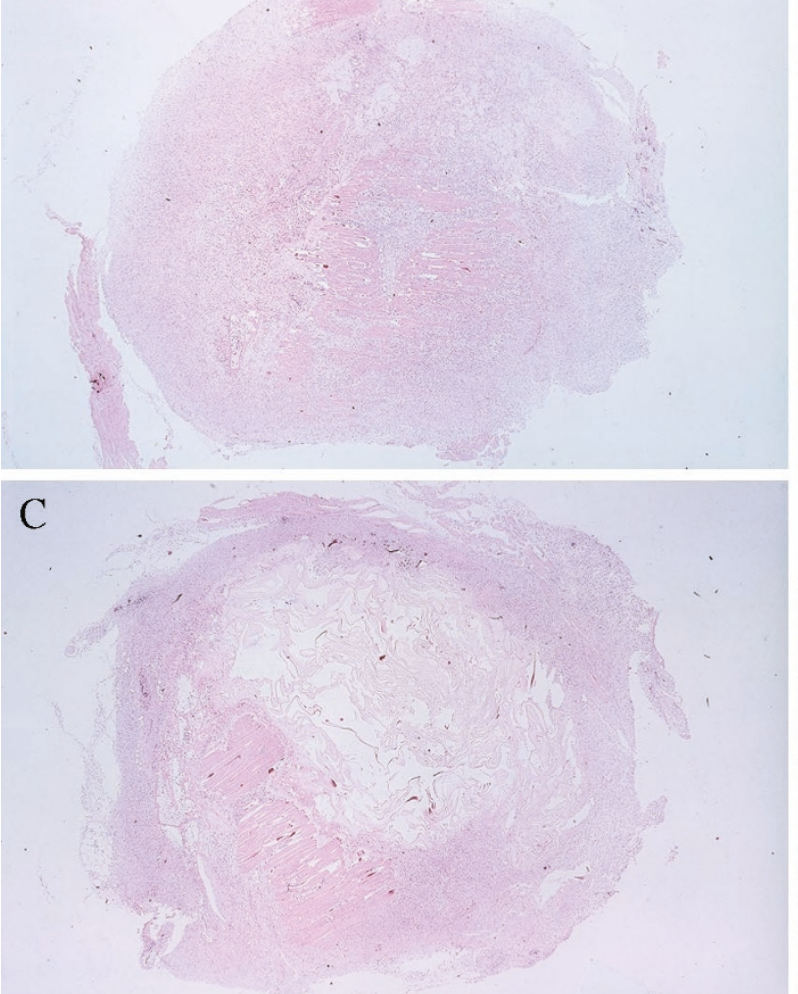

E

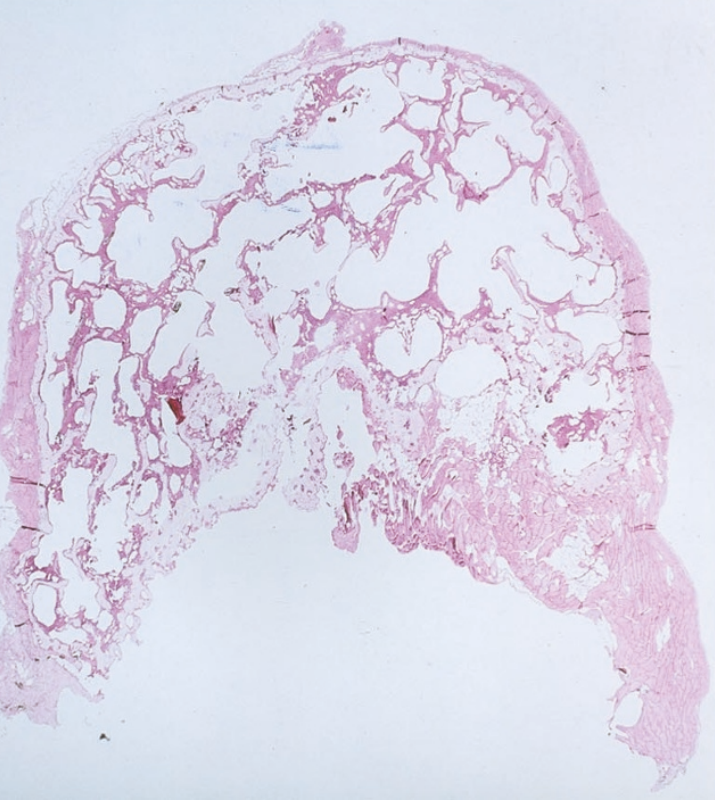

B

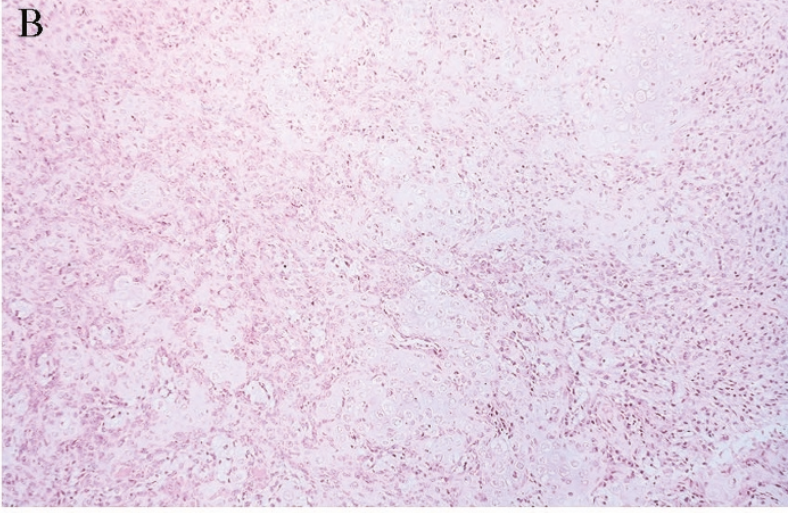

b

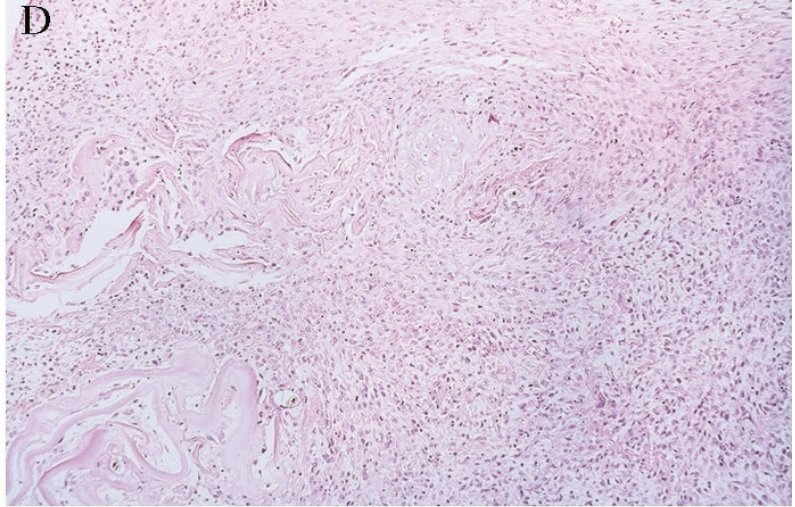

F

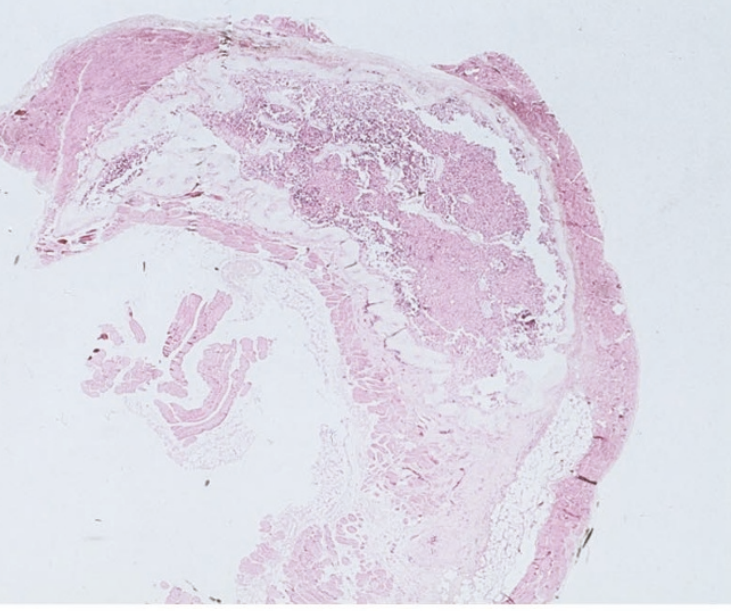

Figure 3.

Histological findings on the ectopic bone induced by BMP-2. Photomicrographs of the cross-sections of BMP-2/atelocollagen pellets in control (A and B) and G-CSF mice ( $C$ and $D$ ) at one week after implantation, and in control $(E)$ and granulocyte colony-stimulating factor (G-CSF) (F) mice at 3 weeks after implantation. Original magnifications: $A$ and $C, \times 40$; $B$ and $D: \times 200$; $E$ and $F: \times 40$. 
the ectopic bone of G-CSF mice by polarized light microscopy (data not shown).

\section{Calcium Content of Induced Tissue}

As shown in Figure 4, the calcium contents of the ectopic bone of G-CSF mice were reduced to $56.2 \%$ of those of controls. There was a statistical significance between these data.

\section{Expression of Type I-Collagen, Osteopontin, and Osteocalcin in the Ectopic Bone}

Semiquantitative reverse transcription-polymerase chain reaction (RT-PCR) analysis of the ectopic bone at 3 weeks disclosed no significant differences in mRNA level of type I-collagen, osteopontin, and osteocalcin between G-CSF mice and control mice (Fig. 5). Next, we determined the expression of these bone-related proteins by immunohistochemistry. These expression patterns of type I-collagen and osteopontin were the same in G-CSF mice and control mice (data not shown). Osteocalcin was positively stained in osteoblasts and their surrounding matrix in the control ectopic bone at 3 weeks (Fig. 6). Interestingly, osteocalcin was positively stained in osteoblasts but not in the matrix in mutant ectopic bone at 3 weeks. Immunohistochemical staining of osteocalcin did not differ in ectopic bone at 6 weeks or in skeletal bones between control and mutant mice (data not shown).

Osteoblastic cells along endosteal bone surface positive for alkaline phosphatase immunoreactivity were counted and expressed as cell number per millimeter (Fig. 7). This indicated that significant reduction in the number of osteoblastic cells in the

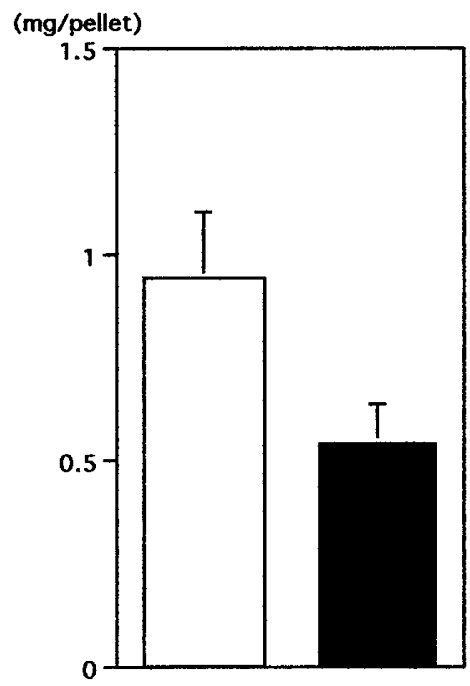

control G-Tg

Figure 4.

Calcium contents of BMP-2/athelocollagen pellets in G-CSF and control mice at 3 weeks after implantation.

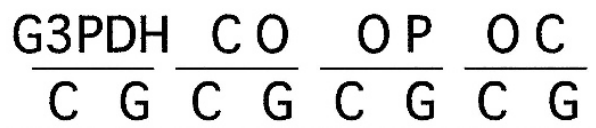

500BP-

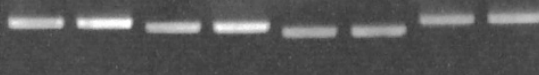

Figure 5 .

Semiquantative reverse transcription-polymerase chain reaction (RT-PCR) analysis of $\alpha 1(\mathrm{I})$ procollagen (CO), osteopontin (OP), and osteocalcin (OC) of the BMP-2/athelocollagen pellet. C, control mice; G, G-CSF mice.

BMP-pellets of G-CSF mice $(9.1+/-3.2$ cells $/ \mathrm{mm})$ compared with those in the control mice $(17.8+/-4.1$ cells $/ \mathrm{mm})(p<0.01)$ (Fig. 7C).

\section{Infiltration of Neutrophils in the BMP-2/Atelocollagen Pellets}

At 1 day after implantation, the BMP-2/atelocollagen pellets were prepared for histological examination. Apparent neutrophilic infiltration was observed, irrespective of the type of mice (Fig. 8). The implant was in the form of button-like plaque and consisted of the collagen matrix and an irregular fibrin network with enmeshed polymorphonuclear leukocytes. The morphology and the number of infiltrating neutrophils were comparable between G-CSF mice and control mice.

\section{BMP-2 Content in the Implants during Ossicle Formation}

On Days 1, 3, 5, 7, 9, and 11 of implantation, pellets were excised and prepared for immunoblotting to quantify BMP in each pellet. BMP immunoreactivity was then compared by densitometric analysis (Fig. 9). In the control mice, the half-life of BMP-2 immunoreactivity occurred between the third and fifth day of implantation. This was prolonged in the G-CSF mice to Day 7 or even later (Day 9).

\section{In Vitro Chondrogenesis of Calvarial Fibroblastic Cells}

The calvarial fibroblastic cells were prepared for in vitro chondrogenesis by BMP-2 in the presence or absence of G-CSF (Fig. 10). On Day 21 of culture, alcian blue-positive cells were counted. The concentration of G-CSF was $10 \mathrm{ng} / \mathrm{ml}$. This is 10 to 20 times higher than the concentration of G-CSF found in the G-CSF mice (Yamada et al, 1996). This experiment revealed that in vitro chondrogenesis using neonatal calvarial cells by BMP-2 was not influenced by the presence of G-CSF.

\section{Discussion}

In this report, we have shown that ectopic bone formation induced by BMP-2 was impaired in transgenic mice overproducing G-CSF. In G-CSF mice, induction of mesenchymal cells and chondrogenesis diminished at an early stage of ectopic bone formation. The lamellar structure was immature, and the 

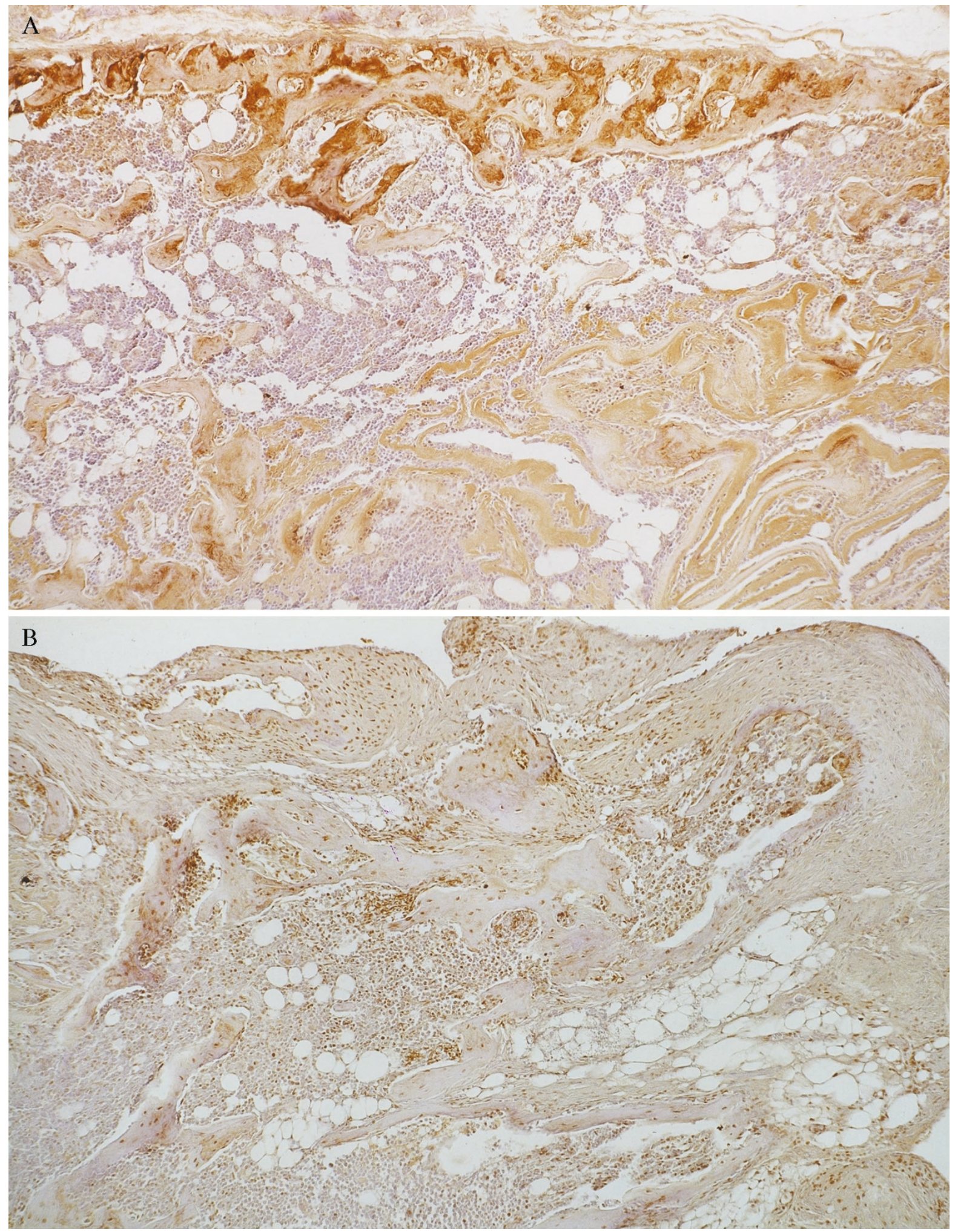

Figure 6.

Immunohistochemical study of osteocalcin in the BMP-2/atelocollagen pellet 3 weeks after implantation. Osteocalcin is positively stained both in osteoblasts and surrounding matrix in control ectopic bone (A). Expression of osteocalcin is restricted in osteoblasts in the ectopic bone of G-CSF mice (B). Original magnification, $\times 100$.

calcium content was decreased in the ectopic bone of G-CSF mice 3 weeks after the implantation of BMP-2. There were no significant differences in mRNA expres- sion levels of type I-collagen, osteopontin, and osteocalcin in the ectopic bone between G-CSF mice and their littermate controls. However, the expression of 

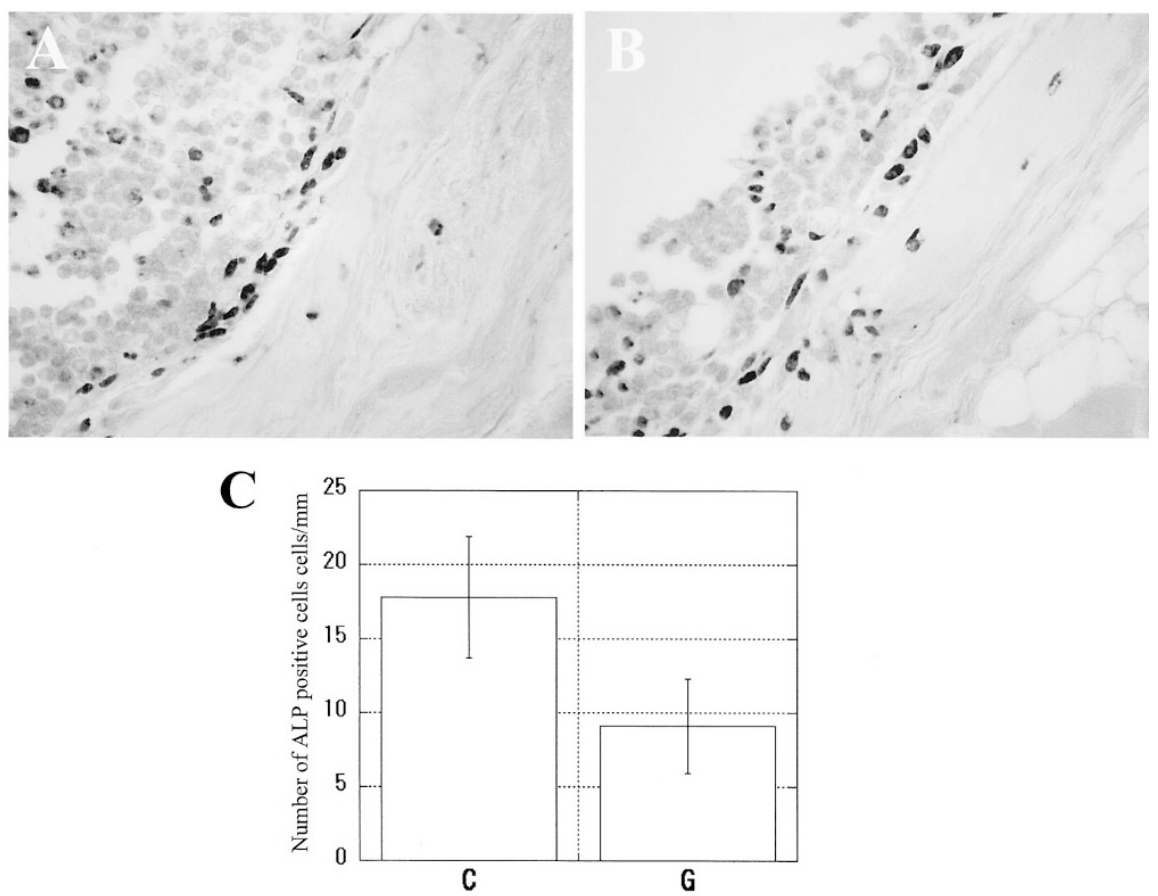

\section{Figure 7.}

Number of alkaline phosphatase-positive cells along endosteal surface of the BMP-2/atelocollagen pellet. Alkaline phosphatase-positive cells along endosteal surface were counted as osteoblastic cells and expressed as cell number/mm of endosteal surface. This criteria omitted alkaline phosphatase-positive hematopoietic cells in the marrow. An apparent reduction in the number of osteoblastic cells was observed in the pellets of the G-CSF mice $(9.1+/-3.2$ cells $/ \mathrm{mm})$ compared with those of the control mice $(17.8+/-4.1)(n=6, p<0.01)$

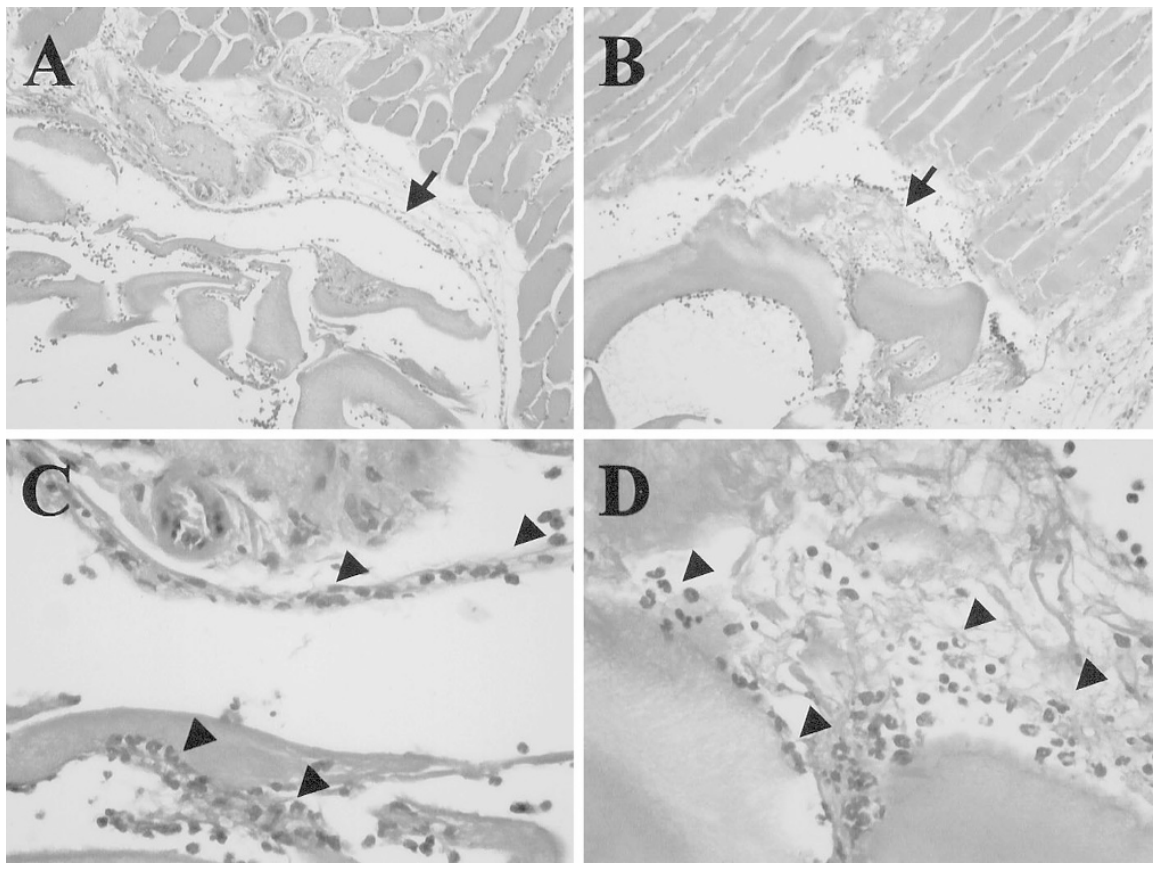

\section{Figure 8.}

Neutrophilic infiltration in the BMP-2/athelocollagen pellet. At Day 1 after implantation, the BMP-2/atelocollagen pellets were prepared for hematoxylin/eosin stain (A and B: G-CSF mice; C and D: control) (original magnification: A and C, $\times 100$; B and D, $\times 400$ ). For pellets in both the G-CSF mice and control mice, neutrophilic infiltration was observed in the collagen bundles as well as adjacent fibrin mesh.

osteocalcin protein in the ectopic bone matrix was less in G-CSF mice than that in littermates. These observations suggest that overexpression of G-CSF impairs in vivo induction of ectopic bone formation by delaying the initial differentiation process of osteoblasts.

In this model, the process of bone formation mimics the heterotopic bone formation described by Reddi 

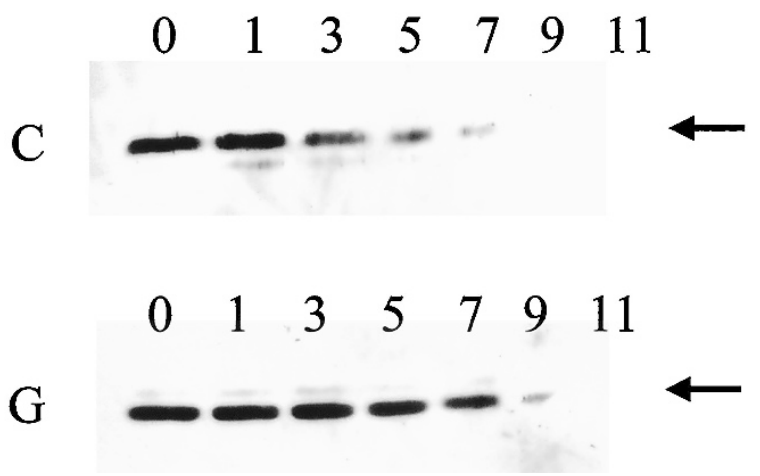

Figure 9.

BMP-2 contents in the BMP-2/athelocollagen pellets during ossicle formation. Immunoblotting to detect BMP-2 reactivity was studied on the BMP-2/ atelocollagen pellets at 1, 3, 5, 9, and 11 days after implantation. Immunoreactivity was reduced gradually during implantation. Densitometric analysis revealed that the half-life of BMP-2 was prolonged to 7 to 9 days in the G-CSF mice, whereas it was 3 to 5 days in the control mice. Numbers indicate the number of days after implantation of BMP-2/atelocollagen pellets ( 0 means the BMP-2/atelocollagen pellet was not implanted as a control of $100 \%$ BMP-2 content).

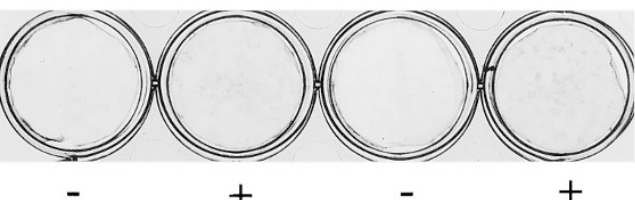

\begin{tabular}{lcccc} 
BMP-2 & - & + & - & + \\
G-CSF & - & - & + & + \\
\hline colony $^{\mathrm{a}}$ & 0.17 & $42.5^{\mathrm{b}}$ & 0.00 & $40.1^{\mathrm{c}}$ \\
$\mathrm{SD}$ & 0.17 & 1.5 & 0.20 & 0.87 \\
\hline
\end{tabular}

a: number of alcian blue positive colonies

b: $p<0.001$ to BMP-2(-) culuture

c: G-CSF treatment did not change number of alcian blue colonies significantly.

$\mathrm{d}$ : number $=6$

\section{Figure 10.}

In vitro chondrogenesis of clavarial fibroblastic cells by BMP-2 in the presence of G-CSF. The calvarial fibroblastic cells obtained from postnatal Days 2 to 5 were cultured in the presence of BMP-2 to induce alcian blue chondroblastic cells with or without G-CSF. The number of chondroblastic cells was determined by deep alcian blue staining with a morphology of hypertrophic chondrocytes under microscope (data not shown).

(1981). Following implantation of the BMP-2 pellet, BMP-2 stimulates the migration of mesenchymal stem cells, which subsequently differentiate into cartilage and bone-forming cells (Schmitt et al, 1999; Urist 1965). Within 1 week, cartilage formation is present and calcification of cartilage begins. Ossification and development of bone marrow results in a fully functioning ossicle of mature heterotopic bone. What steps or stages of enchondral bone formation are impaired in G-CSF mice? Histological examination of the BMP-pellets in G-CSF mice indicated the following: (a) a decreased amount of mesenchymal cell induction at 1 week after implantation, (b) a decreased number and size of the cartilage islands, and (c) a decreased number of osteoblastic cells detected by alkaline phosphatase. Expression of $\alpha 1$ (I)procollagen, osteopontin, and osteocalcin mRNA was not impaired in G-CSF mice. In vitro study indicated that G-CSF has no effect on the BMP-2-induced chondrogenesis. These observations collectively suggest that induction of the mesenchymal stem cells by BMP-2 is impaired in G-CSF mice. By contrast, the further differentiation of mesenchymal stem cells into chondrocytes or osteoblast/osteocytes is not affected. This quantitative decrease of mesenchymal cell induction may be essential for the impairment of bone formation in G-CSF mice.

One might suspect that increased granulocyte would degrade the BMP-2 and result in impaired bone formation (Landesman and Reddi, 1989). However, this hypothesis appears unlikely. First, our histological study showed that there is no significant difference between the mutant mice and littermate controls in the number and morphology of granulocytes infiltrating into the implant. Second, the half-life of BMP-2 contained in the implant estimated by Western blotting is even prolonged in G-CSF mice compared with littermate controls.

It is interesting that immunostaining of osteocalcin decreased in the ectopic bone matrix of G-CSF mice. There were, however, no significant differences in the mRNA expression levels of $\alpha 1$ (l) procollagen, osteopontin, and osteocalcin between these and control mice. Osteocalcin is the major noncollagenous protein of bone produced mainly by osteoblasts. (Hauschka et al, 1989). The decreased numbers of osteoblasts without impairment of differentiation can explain this dissociation.

Why is the initial induction of osteoblasts retarded by the overexpression of G-CSF? Hematopoietic stem cells in bone marrow must provide a continuous source of progenitors for red cells, platelets, monocytes, granulocytes, and lymphocytes (Dexter et al, 1977, To et al, 1997). Bone marrow also contains cells that meet the criteria for stem cells of nonhematopoietic tissues (Prockop, 1997). These stem-like cells for nonhematopoietic tissues, which are referred to as mesenchymal stem cells, have the ability to differentiate into osteoblasts, chondroblasts, adipocytes, and myoblasts. The bone marrow of G-CSF mice is filled with large numbers of cells of hematopoietic lineages, especially neutrophils (Yamada et al, 1996). It is possible that overproduction of G-CSF decreases the relative population of mesenchymal stem cells in bone marrow. This may impair the induction of mesenchymal cells by BMP-2, resulting in the retarded differentiation of osteoblasts.

We previously reported that the characteristics of the ectopic bone induced by BMP mimic those of host bone (Yoshikawa et al, 1998). The sequential gene expression pattern of bone-related proteins in the ectopic bone is comparable to that in embryonic osteogenesis (Hirota et al, 1994). Therefore, investigation of the ectopic bone induced by BMP-2 could provide valuable information about the role of G-CSF in bone formation during development of embryonic bones. The osteoporosis of G-CSF mice is possibly 
mediated by impairment of embryonic bone formation, especially in its early stage.

Severe osteoporosis in the G-CSF transgenic mice provides a number of experimental systems to elucidate mechanisms of, not only bone metabolism, but also a potentially intimate interaction between mesenchyma and hematopoiesis. It is often experienced in the clinical setting that hypoplasia of bone marrow is loosely associated with aged osteoporosis. The coexistence of up-regulated hemopoiesis and osteoporosis in this transgenic mouse may provide a fundamental insight into bone marrow as a source for two independent stem cell systems.

\section{Materials and Methods}

\section{Mice}

The production and maintenance of G-CSF transgenic mice (G-CSF mice) were described previously (Takahashi et al, 1996; Yamada et al, 1996). Transgenic heterozygous mice were examined and nontransgenic littermates were saved as age and sex-matched, wildtype controls for all experiments. All the animals were kept under specific pathogen-free conditions and treated in accordance with guidelines by the Animal Care and Use Committee of Sapporo Medical University.

\section{Preparation and Implantation of BMP-Collagen Composite Pellets}

A BMP/atelocollagen pellet containing $2.5 \mu \mathrm{g}$ of rhBMP-2 (kindly provided by the Genetic Institute, Cambridge, Massachusetts, through the Yamanouchi Pharmaceutical Company, Tokyo, Japan) and $2.4 \mathrm{mg}$ of atelocollagen (Nitta Gelatin, Osaka, Japan) was implanted into a dorsal subfascial pocket in 6-weekold G-CSF mice and littermate mice (Mori et al, 1998). The animals were anesthetized and euthanized 1 day through 3 weeks after implantation of the pellets. The pellets were recovered immediately after death for further investigation.

\section{Morphological Examination of the BMP-Pellets}

Recovered pellets were photographed and radiologically examined with soft $x$-ray (Sofron Type SRO-M50; Soken, Tokyo, Japan). Then the tissues were fixed with $10 \%$ formaldehyde/PBS for 24 hours, decalcified in $5 \%$ formic acid, and embedded in paraffin. The $5-\mu \mathrm{m}$ sections were stained with hematoxylin and eosin. The tissues were also examined immunohistochemically with a rat monoclonal antibody against mouse osteopontin 2.3 (kindly provided by Prof. T. Uede, Institute of Immunological Science, Hokkaido University, Sapporo, Japan), a goat antibody against mouse osteocalcin (Biomedical Technologies, Stoughton, Massachusetts), and a goat antibody for calf alkaline phosphatase (DAKO, Tokyo, Japan). The immunohistochemical staining was done using the avidin-biotin-peroxidase complex (ABC) technique (Histofine SAB-PO kit; Nichirei, Tokyo, Japan).

\section{Measurement of Calcium Content of the BMP-Pellets}

The BMP-pellets were decalcified with $1 \mathrm{ml}$ of $0.6 \mathrm{M}$ $\mathrm{HCl}$ for 24 hours, and the calcium content of $\mathrm{HCl}-$ supernatants was measured using the Calcium C-test (Wako Pure Chemicals, Osaka, Japan).

\section{RT-PCR}

To investigate in vivo levels of mRNA, semiquantitative RT-PCR was performed within the exponential phase of the amplification. Total RNA $(1 \mu \mathrm{g})$ extracted from BMPpellets was reverse transcribed using MuLV reverse transcriptase (Perkin-Elmer, Norwalk, Connecticut) with a random hexamer (Perkin-Elmer), and 5\% of the reaction mixture was amplified with rTaq DNA polymerase (TakaraShuzo, Kyoto, Japan) using the specific primer pairs: 5'-TCTCCACTCTTCTAGTTCCT-3' and 5'-TTGGGTCATTTCCACATGC-3' for $\alpha 1$ (I) procollagen; 5'-ACACTTCACTCCAATCGTCC-3' and 5'-TGCCCTTCCGTTGTTCTCC-3' for osteopontin; 5'-TCTGACAAAGCTTCATGTCC-3' and 5'-AAATAGTGATAGATGCG-3' for osteocalcin; 5'ACTTTGTCAAGCTCATTTCC-3' and 5'-TGCAGCGAACTTTATTGATG-3' for G3PDH. Up to 30 cycles of amplification were performed with a Perkin-Elmer PCR Thermal Cycler at $96^{\circ} \mathrm{C}$ for 15 seconds, at $55^{\circ} \mathrm{C}$ for 10 seconds, and at $72^{\circ} \mathrm{C}$ for 60 seconds.

\section{Quantification of BMP-2 Immunoreactivity in BMP-Pellets}

BMP-pellets were isolated at 1, 3, 5, 7, 9, and 11 days after implantation and stocked frozen. The pellets were thawed and homogenized by dounce homogenizer and extracted by $500 \mu$ of $\times 1$ SDS sample buffer for 2 hours and boiled for 2 minutes. Insoluble debris was omitted by centrifugation and subjected for SDSPAGE followed by Western blotting. Antibody used were goat antibody against recombinant protein corresponding to the amino terminus of the mature, fully processed protein of BMP-2 of human origin (Santa Cruz Biotechnology, Santa Cruz, California). Densitometric analysis was performed on computer-captured images by NIH Image (National Institutes of Health, Bethesda, Maryland), an image processing and analysis program.

\section{In Vitro Chondrocytic Differentiation by rhBMP-2}

In vitro chondrocytic differentiation by rhBMP-2 was conducted as previously described (Kobayashi et al, 2000). In brief, the anterior regions of calvaria (cartilage-free calvaria) from postnatal Days 2 to 5 were incubated with $0.2 \%$ collagenase at $37^{\circ} \mathrm{C}$, and cells were plated for 3 to 4 days in $\alpha$ minimum essential medium ( $\alpha$-MEM) containing 10\% FCS. The cells were plated at $1 \times 10^{4}$ cells $/ \mathrm{cm}^{2}$ on 12 -well plates and cultured in the same medium until confluence. The media were then changed to $\alpha$-MEM containing $10 \%$ FCS, $10 \mathrm{~mm} \beta$-glycerophosphate, 50 $\mu \mathrm{g} / \mathrm{ml}$ ascorbic acid, and $100 \mathrm{ng} / \mathrm{ml}$ of rhBMP-2 in the presence or absence of rhG-CSF at $10 \mathrm{ng} / \mathrm{ml}$. Cells were cultured for 14 to 21 days and fixed by $10 \%$ formaldehyde for 5 minutes. Cells were stained with 
$0.1 \%$ alcian blue in $0.1 \mathrm{~N} \mathrm{HCl}$ overnight, and the number of cartilage nodules was counted under microscope as a deep alcian blue staining with a morphology of hypertrophic chondrocytic cells.

\section{Statistical Methods}

The mean response of each experimental group was compared with its simultaneous control by the unpaired Student's $t$ test. Analysis of variance was used to compare the mean responses of the experimental and control groups.

\section{References}

Bishop NJ, Williams DM, Compston JC, Stirling DM, and Prentice A (1995). Osteoporosis in severe congenital neutropenia treated with granulocyte colony-stimulating factor. $\mathrm{Br} \mathrm{J}$ Haematol 89:927-928.

Dexter TM, Allen TD, and Lajtha LG (1977). Conditions controlling the proliferation of hematopoietic stem cells in vitro. J Cell Physiol 91:335-344.

Glaspy JA and Golde DW (1992). Granulocyte colonystimulating factor (G-CSF): Preclinical and clinical studies. Semin Oncol 19:386-394.

Hauschka PV, Lian JB, Cole DE, and Gundberg CM (1989). Osteocalcin and matrix Gla protein: Vitamin K-dependent protein in bone. Physiol Rev 69:990-1047.

Hirota S, Takaoka K, Hashimoto J, Nakase T, Takemura T, Morii E, Fukuyama A, Morihana K, Kitamura Y, and Nomura S (1994). Expression of mRNA of murine bone-related proteins in ectopic bone induced by murine bone morphogenetic protein-4. Cell Tissue Res 277:27-32.

Kobayashi H, Gao Y-H, Ueta C, Yamaguchi A, and Komori T (2000). Multilineage differentiation of Cbfa1-deficient clavarial cells in vitro. Biochem Biophys Res Com 273: 630636.

Landesman R and Reddi AH (1989). In vivo analysis of the half-life of the osteoinductive potential of demineralized bone matrix using diffusion chambers. Calcif Tissue Int 45:348353.

Lee MY, Fukunaga R, Lee TJ, Lottsfeldt JL, and Nagata S (1991). Bone modulation in sustained hematopoietic stimulation in mice. Blood 77:2135-2141.

Mori S, Yoshikawa H, Hashimoto J, Ueda T, Funai H, Kato M, and Takaoka K (1998). Antiangiogenic agent (TNP-470) inhibition of ectopic bone formation induced by bone morphogenetic protein-2. Bone 22:99-105.

Prockop DJ (1997). Marrow stromal cells as stem cells for nonhematopoietic tissues. Science 276:71-74.
Reddi AH (1981). Cell biology and biochemistry of enchondral bone development. Coll Res 1:209-226.

Schmitt JM, Hwang K, Winn SR, and Hollinger JO (1999). Bone morphogenetic protein: An update on basic biology and clinical relevance. J Orthop Res 17:269-278.

Soshi S, Takahashi HE, Tanizawa T, Endo N, Fujimoto R, and Murota K (1996). Effect of recombinant granulocyte colonystimulating factor (rhG-CSF) on rat bone: Inhibition of bone formation at the endosteal surface of vertebra and tibia. Calcif Tissue Int 58:337-340.

Suzuki M, Adachi K, Sugimoto T, Nakayama H, and Doi K (1999). The development of bone changes induced in rats by recombinant human granulocyte colony-stimulating factor is suppressed by bisphosphonate. Histol Histopathol 14:679686 .

Takahashi T, Wada T, Mori M, Kokai Y, and Ishii S (1996). Overexpression of the granulocyte colony-stimulating factor leads to osteoporosis in mice. Lab Invest 74:827-834.

Takamatsu Y, Simmons PJ, Moore RJ, Morris HA, To LB, and Levesque J-P (1998). Osteoclast-mediated bone resorption is stimulated during short-term administration of granulocyte colony-stimulating factor but is not responsible for hematopoietic progenitor cell mobilization. Blood 92:3465-3473.

To LB, Haylock DN, Simmons PJ, and Juttner CA (1997). The biology and clinical uses of blood stem cells. Blood 89:22332258.

Urist MR (1965). Bone: Formation by autoinduction. Science 150:893-899.

Wada T, Isu K, Takeda N, Usui M, Ishii S, and Yamawaki S (1996). A preliminary report of neoadjuvant chemotherapy NSH-7 study for osteosarcoma: Salvage preoperative chemotherapy based on clinical tumor response and the use of granulocyte-colony stimulating factor. Oncology 53:221227.

Yakisan E, Schirg E, Zeidler C, Bishop NJ, Reiter A, Hirt A, Riehm H, and Welte K (1997). High incidence of significant bone loss in patients with severe congenital neutropenia (Kostmann's syndrome). J Pediatr 131:592-597.

Yamada T, Kaneko H, Izuka K, Matsubayashi Y, Kokai Y, and Fujimoto J (1996). Evaluation of lymphocyte and hematopoietic stem cell numbers in mice transgenic for human granulocyte CSF. Lab Invest 74:384-394.

Yoshikawa H, Taniguchi S, Yamamura H, Mori S, Sugimoto M, Miyado K, Nakamura K, Nakao K, Katsuki M, Shibata N, and Takahashi K (1998). Mice lacking smooth muscle calponin display increased bone formation that is associated with enhancement of bone morphogenic protein responses. Genes Cells 3:685-695. 